\title{
Impact of Automation on Drivers' Performance in Agricultural Semi-Autonomous Vehicles
}

\author{
B. Bashiri, D. D. Mann
}

ABSTRACT. Drivers' inadequate mental workload has been reported as one of the negative effects of driving assistant systems and in-vehicle automation. The increasing trend of automation in agricultural vehicles raises some concerns about drivers' mental workload in such vehicles. Thus, a human factors perspective is needed to identify the consequences of such automated systems. In this simulator study, the effects of vehicle steering task automation (VSTA) and implement control and monitoring task automation (ICMTA) were investigated using a tractor-air seeder system as a case study. Two performance parameters (reaction time and accuracy of actions) were measured to assess drivers' perceived mental workload. Experiments were conducted using the tractor driving simulator (TDS) located in the Agricultural Ergonomics Laboratory at the University of Manitoba. Study participants were university students with tractor driving experience. According to the results, reaction time and number of errors made by drivers both decreased as the automation level increased. Correlations were found among performance parameters and subjective mental workload reported by the drivers.

Keywords. Automatic steering, Driving performance, Level of automation, Mental workload, Reaction time.

$\mathrm{T}$ There is an increasing trend of in-vehicle automation in the agricultural industry. This is mainly for increasing productivity (Edan et al., 2009) and reducing the operator's physical workload. It is difficult to generalize the application of automation in agricultural vehicles due to the diversity of functions that these machines are intended to complete. These automated systems change the nature of the driving task in such vehicles. An automatic steering system, for instance, replaces the physical task of steering (i.e., hands physically turning the steering wheel) with a supervisory task (i.e., checking to ensure that the vehicle's path is acceptable). It has been stated that, besides the benefits, automated driving tasks also introduce new problems for the human operator (Stanton and Marsden, 1996). For example, inadequate mental workload is one of the negative effects of automated systems.

Mental workload reflects the amount of cognitive capacity required to perform a given task (Di Stasi et al., 2013). When the cognitive load for a task is either too high or too low, this is referred to as inadequate mental workload. When applied to driving tasks, drivers may experience imperfect perception, insufficient attention, and inadequate information processing (Brookhuis and de Waard, 2010), leading to reduced human per-

Submitted for review in September 2014 as manuscript number JASH 10977; approved for publication by the Ergonomics, Safety, \& Health Community of ASABE in January 2015.

The authors are Behzad Bashiri, ASABE Member, Graduate Student, and Danny D. Mann, ASABE Member, Professor and Head, Department of Biosystems Engineering, University of Manitoba, Winnipeg, Manitoba, Canada. Corresponding author: Danny Mann, E2-376 EITC, University of Manitoba, Winnipeg, MB Canada R3T 5V6; phone: 204-474-7512; e-mail: Danny.Mann@umanitoba.ca.

Journal of Agricultural Safety and Health 
formance and consequently "reduced traffic safety" (Heijer et al., 1998). In the driving context, controversial effects of automation on the mental workload of drivers have been reported. Various studies have shown that automation of driving tasks of on-road vehicles can result in either increased mental workload (Stanton et al., 2001) or decreased mental workload (Young and Stanton, 1997). Either of these situations can lead to a negative outcome, which might range from a minor collision with no injury to the driver to a fatal crash; thus, case-specific studies are needed to identify the consequences of automation.

Different models have been developed to investigate the effects of automation on human performance (Endsley and Kaber, 1999; Parasuraman et al., 2000; Proud et al., 2003). Common to these models is the assumption that a task can be broken down into information processing subtasks. Furthermore, different levels of automation can be applied to these information processing subtasks. For example, the four-stage model proposed by Parasuraman et al. (2000) that has been widely used as a starting point in automation studies (de Tjerk et al., 2010) breaks a task down into (1) information acquisition, (2) information analysis, (3) decision and action selection, and (4) action implementation subtasks. Depending on the automated system, different levels of automation, from none to high, can be defined for each of these subtasks. After this process, various human performance measures, such as mental workload measures, can be used for selecting an optimal involvement of human operators in automated work environments.

For the measurement of mental workload, various methods have been used in different domains. These methods can be categorized as (1) subjective reports, (2) task performance measures, and (3) physiological measures (Brookhuis et al., 2009; Desai, 1993; Veltman and Gaillard, 1993). Subjective reports include various questionnaires for collecting feedback from subjects or observers. Task performance measures include techniques for direct measurement of operator behavior while performing a task. Physiological measures are based on the body's physical response to changes in mental demand (i.e., heart rate variability and pupil diameter).

Task performance measures in the driving context are used to assess the ability of drivers to perform tasks accurately on a time-limited basis. The goal is direct assessment of the operator's ability to perform the driving task at an acceptable level (Brookhuis et al., 2009). Reaction time and accuracy of actions are two important components of task performance. Degree of automation, task difficulty, and task type can directly affect these components. Improvement in reaction time was reported when drivers were driving with an autonomous control mode in military semi-autonomous vehicles (Gempton et al., 2013). Another experiment by Sethumadhavan (2009) demonstrated the benefits of high levels of automation in multi-task environments where operators had to perform multiple tasks concurrently. Johnson and Widyanti (2011), in their study on cultural influences on the measurement of subjective mental workload, measured reaction time of subjects in response to a hybrid memory/visual search task. They found that reaction time was increased by increasing task difficulty.

Considering the differences that exist between on-road driving and in-field operation, as described by Bashiri and Mann (2013), results from studying on-road vehicles cannot be applied to agricultural vehicles. Identifying the effects of new automated systems on drivers' mental workload in agricultural vehicles demands specific studies. A simulator experiment was performed to examine the effects of task automation in agricultural vehicles on drivers' mental workload. Multiple measurement techniques were used to assess drivers' mental workload. This article focuses on drivers' reaction time and accuracy of 
actions when working with a semi-autonomous machine system (consisting of a tractor and air seeder) with different levels of automation support. The hypothesis was that increasing the automation support level would reduce the reaction time of the operator and increase the accuracy of the operator's actions. The results from a self-assessment technique, reported by Bashiri and Mann (2013), were used to identify any possible correlations between mental workload measures.

\section{Methodology}

\section{Participants}

Research subjects were 30 undergraduate and graduate university students, 28 males and 2 females, who volunteered for participation in the study. The participants ranged in age from 18 to 25 years, and the average age was $20.9 \pm 2$ years. They all had at least one year (season) of tractor driving experience (average of $7.7 \pm 3.9$ years). None of them had prior experience with the current driving simulator; however, one subject had participated in a study with a previous version of the driving simulator. The number of subjects was selected based on a power test.

\section{Apparatus and Tasks}

The tractor driving simulator (TDS) located in the Agricultural Ergonomics Laboratory at the University of Manitoba was used in this study. Various agricultural vehicles and implements can be simulated by this TDS. The layout of the simulator is shown in figure 1. It includes an actual tractor cab, a curved screen, and multiple video projectors. In addition, two video monitors are located behind the cab for simulating agricultural implements. A monitor inside the cab is used to display implement information to the driver. In addition to the implement parameters, a mapping system is provided to assist drivers with locating the tractor in the field. In this study, a machine system consisting of a tractor and an air seeder was simulated with the TDS.

A tractor-air seeder system is used for planting in agricultural fields. Besides driving functions, such as forward speed and longitudinal and lateral controls, operators are required to monitor and control the air seeder parameters. This includes adjusting the seed and fertilizer application rates, fan rotational speed, and tool working depth; monitoring the levels of seed and fertilizer in the hoppers; and removing any residue or mud that blocks seed and fertilizer application in the seeding units. In current tractor-air seeder systems, automation has been implemented to assist operators with both the vehicle steering task and the implement control and monitoring functions. For this research, the simulator was modified to mimic both vehicle steering task automation (VSTA) and implement control and monitoring task automation (ICMTA). The simulator was programmed to introduce errors at random times because machine performance is not expected to be optimal at all times. Test subjects were expected to monitor the air seeder parameters (provided on the implement information display in the cab) to keep them within specified ranges. Consequently, the simulator drivers were required to monitor and control the air seeder parameters as they were performing the driving function. 

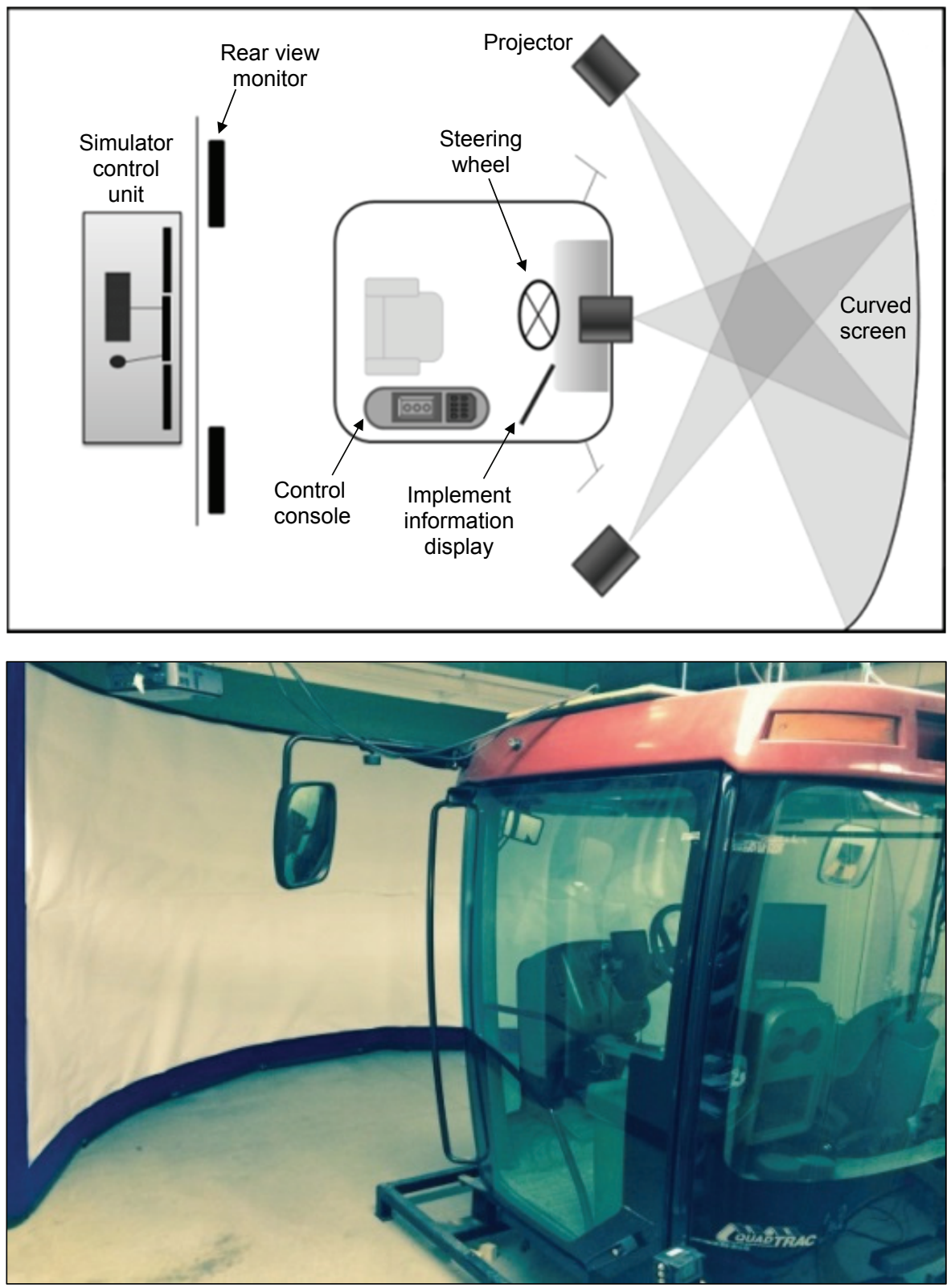

Figure 1. (top) Plan view of the simulator layout and (bottom) photograph of the simulator.

\section{Independent Variables}

According to the functions involved in the operation of a tractor-air seeder system, vehicle steering task automation (VSTA) and implement control and monitoring task automation (ICMTA) were considered the two independent variables of the study. VSTA included two levels: manual steering and automatic steering. ICMTA included five lev- 
els: manual monitoring and control, information acquisition automation, information analysis automation, decision and action selection automation, and action implementation automation.

With respect to VSTA, the automatic steering used in this study represented current auto-steer technology that is being used in many agricultural vehicles. An auto-steer system eliminates the need for operators to steer when the tractor is being operated in straight lines in open fields. It uses GPS data to correct the position of the vehicle in order to avoid gaps and overlaps between paths. In this mode, which is considered the low taskload condition, the control and monitoring of the air seeder was the main task for the operator. However, with manual steering (i.e., high taskload context), operators were expected to perform the vehicle steering task while simultaneously monitoring the status of the air seeder and making control adjustments as required. Automatic steering reflected a pure supervisory task, while manual steering was a combination of physical and supervisory tasks.

With respect to ICMTA, the four-stage model of Parasuraman et al. (2000) was used to define the levels of automation support. In the manual mode, no visual support was added to the information display of the simulator, as previously designed by Karimi et al. (2011). Operators were required to detect errors using the information display and take corrective action using the control console. In the information acquisition mode, the computer was responsible for detecting errors and highlighting them, leaving the remainder of the task to the operator (i.e., taking corrective action using the control console). In the information analysis mode, the computer analyzed the data and made predictions of errors. In this mode, a warning message was provided on the information display; operators were required to interpret the message and perform the necessary action. In the decision and action selection mode, the computer suggested the proper action, requiring the operator to implement the action. Finally, the action implementation mode was the highest level of automation in this study; the computer performed all of the information processing functions and only informed the operator after performing a task. A snapshot of the air seeder information display in information analysis mode, as well as the mapping system, is shown in figure 2. Brief descriptions of the five ICMTA support modes, with sample warning messages, are provided in table 1.

\section{Dependent Variables}

The performance measure was based on the driver's reaction time and the number of errors made. Reaction time was defined as the period of time between the emergence of an error and the time that a driver started to make an adjustment. In terms of human errors, three conditions were specified as failures: (1) if a parameter adjustment was missed or a parameter was adjusted at the wrong time (WT), (2) if a parameter was adjusted in a wrong direction (WD), and (3) if a wrong parameter (WP) was adjusted. For example, if the seed application rate did not require any adjustment but the operator adjusted it anyway, an error was made. If the seed application rate needed to be increased, but the operator mistakenly decreased it or adjusted another parameter, such as fertilizer application rate, this was also considered a failure. It should be noted that not all the failure scenarios were applicable to all the parameters. For instance, in the case of blockage, removing the blockage was the only available option, so adjusting in a wrong direction would not apply to this parameter. The TDS recorded and stored the implement parameters and control 


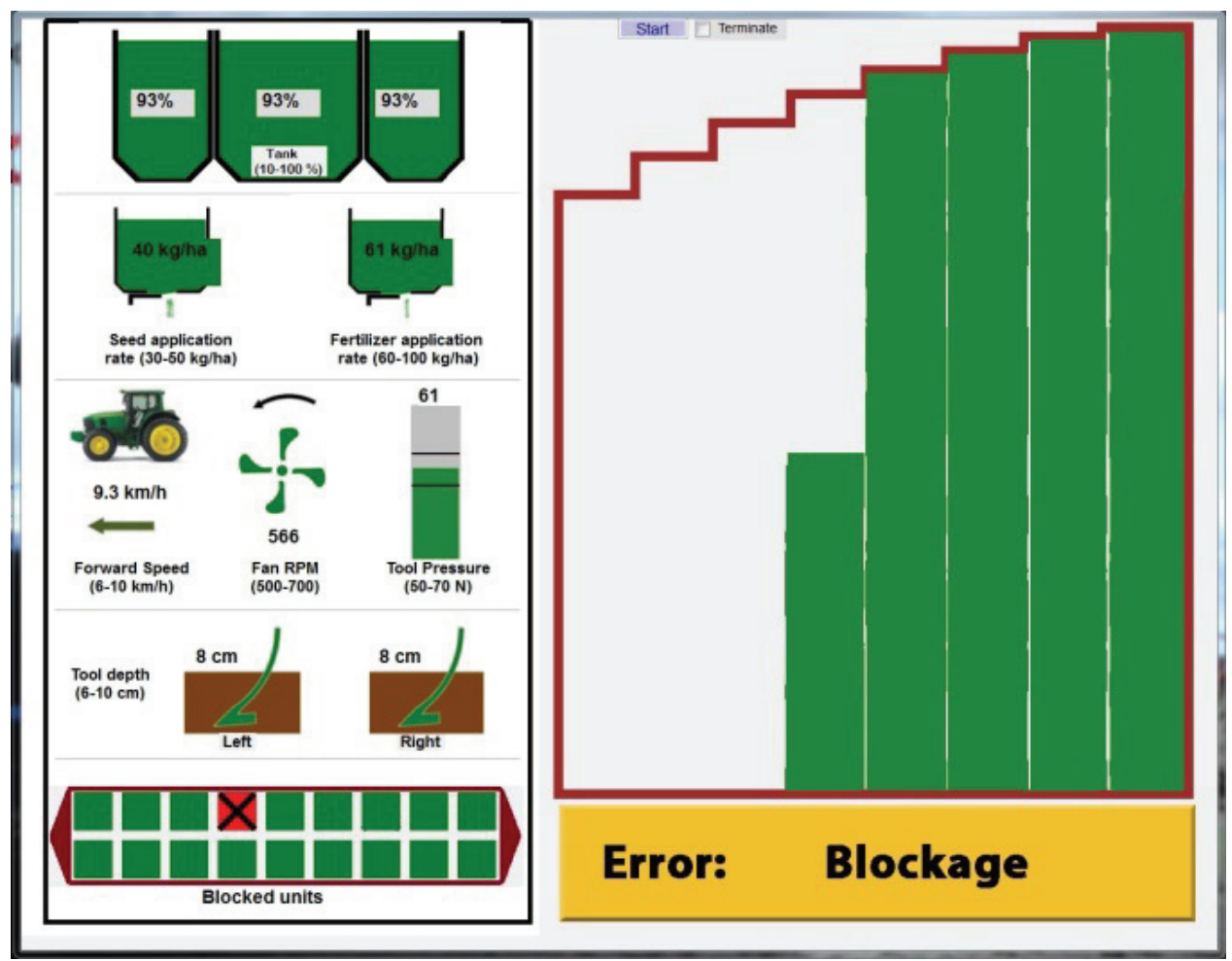

Figure 2. Air seeder information display and mapping system.

Table 1. ICMTA support modes.

\begin{tabular}{ccc}
\hline Support Mode & Description & Message Example \\
\hline Manual & No visual support is provided & N/A \\
\hline Information acquisition & $\begin{array}{c}\text { Computer detects error and } \\
\text { highlights it }\end{array}$ & N/A \\
\hline Information analysis & $\begin{array}{c}\text { Computer analyzes data and } \\
\text { makes predictions }\end{array}$ & Error: tool pressure \\
\hline Decision and action selection & Computer suggests required action & Increase the tool pressure \\
\hline Action implementation & Computer performs the task & The tool pressure is adjusted \\
\hline
\end{tabular}

actions of the drivers. Data from the simulator were reviewed separately for each participant in order to calculate reaction time and to count the number of errors made.

In this experiment, the driving activity load index (DALI) developed by Pauzié (2008) was used for subjective assessment of drivers' mental workload. Using this index, participants were able to rate different aspects of the workload demand of a given task. This included attentional demand, visual demand, stress, temporal demand, and task interference. A global score of mental workload imposed by the task was calculated by averaging the scores of these parameters. For more information on the DALI used in this experiment, refer to Bashiri and Mann (2013).

\section{Experimental Design and Analysis}

A $2($ VSTA $) \times 5($ ICMTA $)$ design was used in the study. VSTA was applied as a be- 
tween-subject design, meaning that half of the participants performed the trial with manual steering and the other half with automatic steering. On the other hand, ICMTA was applied as a within-subject design, so each participant was required to perform the trial with all five of the automation support modes. Arranging the experiments in the form of repeated $5 \times 5$ Latin squares made it possible to avoid the learning effect and to accommodate the limited number of subjects. The experimental design included six $5 \times 5$ Latin squares, sharing the same columns (driving period), with subjects in rows. According to the experimental condition, subjects and driving periods were assumed as blocking factors, acting as random effects.

Appropriate descriptive statistics were calculated for the parameters of the dependent variables. The analyses were performed with linear mixed models using the PROC MIXED procedure in SAS (ver. 9.3, SAS Institute, Inc., Cary, N.C.). Shapiro-Wilk's test was used for normality tests prior to analysis. Data with substantial deviations, which only included reaction time and error, were normalized by means of a logarithmic transformation. Post-hoc differences of least squares means were used to determine the source of any significant effects. The non-parametric Wilcoxon test was used in the rest of the analysis wherein the data distributions were not normal and application of a parametric statistic was not applicable. Statistically significant differences were accepted at the $95 \%$ confidence level $(\mathrm{p}<0.05)$ or greater. All values for parametric analyses are presented as means \pm standard error.

\section{Procedure}

Before the trial began, subjects received explanation of the test procedure and were provided with necessary instructions. They were asked to sign a consent form containing such information. A 15 min training session was administered to make subjects comfortable with the test procedure and allow them to familiarize themselves with the simulator and the implement control console. This session illustrated all of the driving conditions that subjects needed to complete in the trial. After the training session, they completed the main trial, which included five driving blocks of 12 min each. At the end of each driving block, paper-based queries of DALI were given to the subjects. At the end of the trial, each subject was compensated monetarily for volunteering in the experiment.

\section{Results}

\section{Performance}

According to the results, the average reaction time with manual steering was 1.01 $\pm 0.15 \mathrm{~s}$. For automatic steering, this value was $0.67 \pm 0.12 \mathrm{~s}$. By eliminating results for the action implementation mode, in which operators were not involved in the task loop, the average reaction times were $1.96 \pm 0.11 \mathrm{~s}$ and $1.71 \pm 0.12 \mathrm{~s}$ for manual and automatic steering, respectively. Figure 3 shows the means of reaction time for manual and automatic steering for the five automation support modes. The statistical analysis showed no VSTA effect on reaction time $(F(1,28)=1.31, \mathrm{p}=0.26)$.

The statistical analysis showed a significant effect of ICMTA support on reaction time $(\mathrm{F}(4,94)=68.79, \mathrm{p}<0.001)$. According to the differences of least square means, no significant differences were observed between the manual $(2.04 \pm 0.17 \mathrm{~s})$ and information acquisition modes $(2.25 \pm 0.17 \mathrm{~s})$. The information analysis $(1.58 \pm 0.13 \mathrm{~s})$ and decision and action selection $(1.46 \pm 0.12 \mathrm{~s})$ modes had similar effects on reaction time, but with 

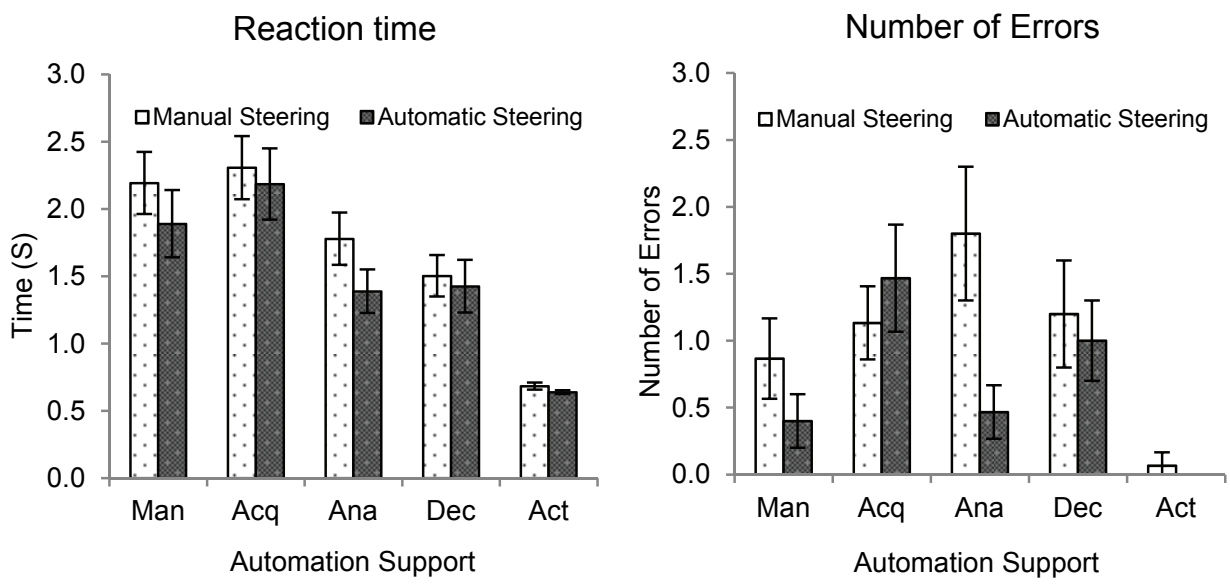

Figure 3. Operators' reaction time and number of errors (mean \pm SEM) in different taskloads and automation support modes: Man $=$ manual, $\mathbf{A c q}=$ information acquisition, Ana $=$ information analysis, Dec $=$ decision and action selection, and Act $=$ action implementation.

values significantly lower than those of the manual and information acquisition modes. The action implementation mode resulted in the lowest reaction time $(0.66 \pm 0.02 \mathrm{~s})$, presenting significant differences with the other automation support modes.

Means of the number of errors that participants made in the different VSTA and ICMTA conditions are also shown in figure 3. In total, operators made 126 errors: 62 errors were related to WT, 58 to WD and 6 to WP. Manual steering resulted in 76 failures, and automatic steering resulted in only 50 failures; however, the difference was not significant $(\mathrm{F}(1,28)=1.7, \mathrm{p}=0.2)$. ICMTA support showed a significant effect on the number of failures $(F(4,108)=13.55, \mathrm{p}<0.001)$. Likewise, the ICMTA-VSTA interaction effect was significant $(\mathrm{F}(4,108)=3.04, \mathrm{p}=0.02)$.

The action implementation mode significantly reduced the number of errors $(0.03$ $\pm 0.03)$. A low number of errors was also observed in the manual mode $(0.63 \pm 0.19)$. Both of these modes showed significant differences with each other and with the rest of the automation support modes. The information acquisition $(1.3 \pm 0.23)$, information analysis $(1.13 \pm 0.29)$, and decision and action selection $(1.1 \pm 0.22)$ modes resulted in similar numbers of errors, showing no significant differences with one another. The ICMTAVSTA interaction was due to the fact that subjects made lower numbers of errors in the information analysis mode while driving with automatic steering $(0.47 \pm 0.24)$ compared to manual steering $(1.8 \pm 0.49)$.

Wilcoxon rank sum analysis showed no effect of steering mode on WD $(z=-0.58, p=$ $0.56)$ and WP $(z=0.82, p=0.41)$. On the other hand, steering mode significantly affected RT $(z=-2.20, p=0.03)$. Based on the results, automatic steering significantly reduced the number of errors pertaining to WT. Kruskal-Wallis tests indicated that automation support mode significantly affected WD $(\mathrm{p}<0.001)$ and WT $(\mathrm{p}<0.01)$ but had no effect on WP $(\mathrm{p}=0.05)$. For WD, a pairwise two-sided multiple comparison analysis using the Dwass, Steel, Critchlow-Fligner (DSCF) method showed that only the action implementation mode resulted in significantly lower values compared to the manual $(\mathrm{z}=-3.2, \mathrm{p}=$ $0.01)$, information acquisition $(\mathrm{z}=5.15, \mathrm{p}<0.001)$, information analysis $(\mathrm{z}=3.2, \mathrm{p}=$ 
Table 2. Correlations among mental workload and performance parameters. ${ }^{\text {[a] }}$

\begin{tabular}{|c|c|c|c|c|c|c|c|}
\hline & Attention & Visual & Stress & Temporal & Interference & Global & $\begin{array}{c}\text { Reaction } \\
\text { Time }\end{array}$ \\
\hline Visual & $0.73 * *$ & - & - & - & - & - & - \\
\hline Stress & $0.43 * *$ & $0.37 * *$ & - & - & - & - & - \\
\hline Temporal & $0.48 * *$ & $0.44 * *$ & $0.76 * *$ & - & - & - & - \\
\hline Interference & $0.40 * *$ & $0.29 * *$ & $0.56 * *$ & $0.59 * *$ & - & - & - \\
\hline Global & $0.79 * *$ & $0.74 * *$ & $0.79 * *$ & $0.82 * *$ & $0.70 * *$ & - & - \\
\hline Reaction time & $0.34 * *$ & $0.18^{*}$ & $0.45 * *$ & $0.51 * *$ & $0.39 * *$ & $0.52 * *$ & - \\
\hline Number of errors & 0.14 & $0.19 *$ & $0.32 * *$ & $0.32 * *$ & $0.27 * *$ & $0.32 * *$ & $0.48 * *$ \\
\hline
\end{tabular}

as Asterisks indicate statistical significance: $* * \mathrm{p}<0.01$ and $* \mathrm{p}<0.05$.

$0.01)$, and decision and action selection $(\mathrm{z}=3.4, \mathrm{p}<0.01)$ modes. For RT, the only significant difference was found between the decision and action selection mode and the action implementation mode $(\mathrm{z}=3.65, \mathrm{p}<0.01)$.

\section{Mental Workload and Performance Correlation}

The Pearson correlation coefficients between the mental workload and performance parameters are shown in table 2. It can be seen that there were positive correlations among all of the parameters. The only non-significant correlation was found between attentional demand and number of errors. Notably strong correlations were observed between attentional demand and visual demand, and between stress and temporal demand. The correlation between attentional demand and visual demand suggests that the subjects paid a great deal of attention to the implement monitoring task and the mapping system using the information display. The strong positive correlation of stress with temporal demand shows that higher timing demand resulted in higher stress levels in the subjects. The strong correlations between the global workload score and its parameters were due to the fact that the global workload score was derived from its parameters. In the case of reaction time, moderate correlations were found with stress and global workload score. The subjects tended to react more slowly when they perceived higher pressure and specific constraint due to timing demand of the whole activity. Furthermore, they gave higher ratings for DALI parameters when they felt higher timing pressure. Number of errors had only a moderate correlation with reaction time. This means that the subjects made more errors when their reaction times were higher, indicating that the later they realized a parameter needed adjustment, the more likely they were to fail.

\section{Discussion}

The hypothesis of this study was that the drivers' performance would increase with an increase in the level of automation. The hypothesis was confirmed in the case of both performance parameters (i.e., reaction time and number of errors). The VSTA effect was not significant, but in the case of ICMTA, increasing automation level was associated with lower reaction time and number of errors. This result confirms the findings of previous studies (Gempton et al., 2013; Sethumadhavan, 2009) by presenting automation benefits on drivers' performance.

The lowest reaction time and number of errors were achieved with the highest level of ICMTA support. In this mode, the automated system was responsible for parameter adjustment. It was expected that the average reaction time would be zero or very close to zero in this mode, but the simulator computer and I/O boards did not allow for such a fast reaction. Furthermore, the manual mode caused a lower number of errors compared to the 
information acquisition, information analysis, and decision and action selection modes. This could be mainly due to each individual's performance when driving the simulator. Some of the subjects made many errors, while others did not fail at all.

Positive correlations among global workload score, reaction time, and number of errors suggest the benefits of automation in agricultural vehicles, yet more studies will be needed to generalize this finding as several limitations likely affected the results. First, variability among subjects could be observed based on the results relating to their skills in performing the trials. Driving experience and the subject's state when driving the simulator could have a significant effect in this case. Another factor could be the time of day when the experiment was completed; subjects could select a morning or afternoon session. In addition, the duration of the training session and driving blocks in the present experimental setup were short compared to the long hours of driving in a field.

\section{Conclusion}

The simultaneous measurement of agricultural vehicle operators' mental workload and performance allows better understanding of the interactions between operators and the automated systems they use. An experiment was performed to assess the effect of vehicle steering task automation (VSTA) and implement control and monitoring task automation (ICMTA) on the tractor driver's driving performance as an indicator of mental workload. Task automation improved driving performance parameters of reaction time and accuracy of actions. The results suggested a greater impact of ICMTA than VSTA.

The results from this research provide some evidence that there are benefits to the use of automation in agricultural machines. However, caution should be exercised before making broad generalizations because these results were obtained in a simulator study without the benefit of highly skilled air seeder operators. Furthermore, there are substantial differences between tractor-air seeder systems and other machine systems used in production agriculture.

\section{Acknowledgements}

The authors acknowledge the financial assistance of the Natural Science and Engineering Research Council of Canada (NSERC). We also thank G. H. Crow, L. Onischuk, H. Khazanehei, and M. Porter for their support, and Case-New Holland for donating the tractor cab used in the development of the driving simulator.

\section{References}

Bashiri, B., \& Mann, D. D. (2013). Drivers' mental workload in agricultural semi-autonomous vehicles. Proc. Human Factors and Ergonomics Soc. Ann. Mtg., 57, 1795-1799. http://dx.doi.org/10.1177/1541931213571402.

Brookhuis, K. A., \& de Waard, D. (2010). Monitoring drivers' mental workload in driving simulators using physiological measures. Accident Analysis and Prevention, 42(3), 898-903. http://dx.doi.org/10.1016/j.aap.2009.06.001.

Brookhuis, K. A., van Driel, C. J. G., Hof, T., van Arem, B., \& Hoedemaeker, M. (2009). Driving with a congestion assistant: Mental workload and acceptance. Appl. Ergonomics, 40(6), 10191025. http://dx.doi.org/10.1016/j.apergo.2008.06.010.

de Tjerk, E. G., Henryk, F. R. A., \& Neerincx, M. A. (2010). Adaptive automation based on an objectoriented task model: Implementation and evaluation in a realistic $\mathrm{C} 2$ environment. J. Cognitive Eng. Decision Making, 4(2), 152-182. http://dx.doi.org/10.1518/155534310X522851. 
Desai, T. P. (1993). Stress and mental workload: A study in an industrial organisation. Indian J. Ind. Relations, 28(3), 258-273.

Di Stasi, L. L., Antolí, A., \& Cañas, J. J. (2013). Evaluating mental workload while interacting with computer-generated artificial environments. Entertainment Computing, 4(1), 63-69. http://dx.doi.org/10.1016/j.entcom.2011.03.005.

Edan, Y., Han, S., \& Kondo, N. (2009). Automation in agriculture. In S. Y. Nof(Ed.), Springer Handbook of Automation (1095-1128). Berlin, Germany: Springer.

Endsley, M. R., \& Kaber, D. B. (1999). Level of automation effects on performance, situation awareness, and workload in a dynamic control task. Ergonomics, 42(3), 462. http://dx.doi.org/10.1080/001401399185595.

Gempton, N., Skalistis, S., Furness, J., Shaikh, S., \& Petrovic, D. (2013). Autonomous control in military logistics vehicles: Trust and safety analysis. In D. Harris (Ed.), Engineering Psychology and Cognitive Ergonomics (Vol. 8020, pp. 253-262). Berlin, Germany: Springer.

Heijer, T., Brookhuis, K., van Winsum, W., \& Duynstee, L. (1998). Automation of the driving task. R-98-9. Leidschendam, The Netherlands: SWOV. Retrieved from http://publicaties.minienm.nl/download-bijlage/35246/automation-of-the-driving-task.pdf.

Johnson, A., \& Widyanti, A. (2011). Cultural influences on the measurement of subjective mental workload. Ergonomics, 54(6), 509-518. http://dx.doi.org/10.1080/00140139.2011.570459.

Karimi, D., Mann, D. D., \& Yan, J. (2011). A comparison of textual, symbolic, and pictorial presentation of information on an air-seeder display. Australian J. Agric. Eng, 2(4), 90-95.

Parasuraman, R., Sheridan, T. B., \& Wickens, C. D. (2000). A model for types and levels of human interaction with automation. IEEE Trans. Syst., Man and Cybernetics A, 30(3), 286-297. http://dx.doi.org/10.1109/3468.844354.

Pauzié, A. (2008). A method to assess the driver mental workload: The driving activity load index (DALI). IET Intelligent Transport Syst., 2(4), 315-322. http://dx.doi.org/10.1049/iet-its:20080023.

Proud, R. W., Hart, J. J., \& Mrozinski, R. B. (2003). Methods for determining the level of autonomy to design into a human spaceflight vehicle: A function specific approach. In Proc. 2003 Performance Metrics for Intelligent Systems (PerMIS) Workshop. Gaithersburg, Md.: NIST.

Sethumadhavan, A. (2009). Effects of levels of automation on air traffic controller situation awareness and performance. PhD diss. Lubbock, Texas: Texas Tech University.

Stanton, N., \& Marsden, P. (1996). From fly-by-wire to drive-by-wire: Safety implications of automation in vehicles. Safety Sci., 24(1), 35-49. http://dx.doi.org/10.1016/S0925-7535(96)00067-7.

Stanton, N., Young, M., Walker, G., Turner, H., \& Randle, S. (2001). Automating the driver's control tasks. Intl. J. Cog. Ergonomics, 5(3), 221-236. http://dx.doi.org/10.1207/S15327566IJCE0503_5.

Veltman, J. A., \& Gaillard, A. W. K. (1993). Indices of mental workload in a complex task environment. Neuropsychobiol., 28(1-2), 72-75. http://dx.doi.org/10.1159/000119003.

Young, M. S., \& Stanton, N. A. (1997). Automotive automation: Investigating the impact on drivers' mental workload. Intl. J. Cognitive Ergonomics, 1(4), 325-336. 